\title{
EL SUJETO TACHADO: UN SUJETO CONSTRUIDO
}

\author{
Cristina MARQUES RODILLA \\ Instituto Ramiro de Maeztu. Madrid
}

Sobre la disolución del sujeto ya nos avisó Foucault de forma provocadora. Lo más triste era aceptar su corta duración. Además de la reciente invención del hombre 1 , su futuro era más que incierto porque lo que se hizo fue igualar 'muerte del hombre' con 'fin del sujeto'. Aunque Freud y Heidegger, entre otros, ya se habían encargado de producir la herida narcisista, de negar la existencia del sujeto autoconsciente, transparente a sí mismo y absoluto en su poder, Foucault y su arqueología propiciaban, quizás sería mejor decir que precipitaban, el fin del humanismo en sus más diversas variantes.

Una vez producida la muerte del hombre cabe buscar el nuevo sujeto en la cibernética y en la sociedad sin hombres defendida por Luhmann, pero cabe también la crítica del sujeto sin su supresión. No hace falta volver al humanismo renacentista ni al historicismo marxista que reescribe el sueño antropológico a partir de la figura del doble que viene a ocupar el lugar vacío, bastaría con la crítica del sujeto porque ésta arroja siempre un "resto", deja siempre un residuo en tanto que proceso de subjetivación.

Nosotros trataremos ese resto como deseo y goce, como singularidad o sí mismo desde la perspectiva de Lacan, pero creemos que ese mismo intento de aprehender la subjetividad escindida está presente en todos los postestructura-

1 «En todo caso una cosa es cierta: que el hombre no es el problema más antiguo ni el más constante que se haya planteado el saber humano. Al tomar una cronologia relativamente breve y un corte geográfico restringido - la cultura europea a partir del siglo xvi- se puede estar seguro de que el hombre es una invención reciente». FoucaulT, M.: Las palabras y las cosas, Barcelona, 1984, p. 375. 
listas franceses, Badiou, Deleuze, Guattari, incluso el mismo Foucault, que apuntan hacia una consideración del sujeto menos sujeto que sujetado. Hay, pues, una «imposibilidad» radical: la que va de la afirmación de un sujeto a la sujeción de tal sujeto en el código.

\section{La estructura y el cero del sujeto}

No vamos a entrar en discusión respecto a la adscripción de Lacan al estructuralismo ${ }^{2}$, pero está claro que desde 1953, en 'El discurso de Roma' ${ }^{3}$, Lacan se adscribe a la lógica del significante para tratar de aprehender al sujeto del inconsciente. En su tesis doctoral (1932) sobre la psicosis hace un intento de aprehensión de ésta por la estructura lingüística que proseguirá su trayectoria hasta que en 1962 se completa con una torsión que le lleva hasta una topología del sujeto, topología que en 1975 culmina con la creación del nudo borromeo.

Sea o no sea Lacan un estructuralista a la letra, su noción de sujeto es correlativa al cero del sujeto tal como Benveniste lo considera. Ese vacío que es el sujeto será ocupado por la estructura lingǘrtica que sujeta al sujeto al código que le introduce en la comunidad de parlêtres ('parlanteseres').

El sujeto tachado, $\$$, de Lacan es ante todo un sujeto mortificado por el lenguaje, dividido por el significante y escindido en el pensamiento. Pero no puede ser sólo eso porque, entonces, ¿qué sentido tendría pasar por el diván? Tampoco se quiere decir que el sujeto deje su sujeción, que pueda escapar a la mortificación significante, se quiere indicar que ese artificio que se denomina

2 No es éste un artículo dedicado a analizar la adscripción de Lacan al estructuralismo, pero sí queremos marcar las distancias teóricas entre Lacan y Lévi-Strauss al menos en dos puntos clave: $10^{\circ}$ ) en cuanto a la afirmación hecha por LEVI-STRAUSS en su Antropología estructural (Eudeba, B. Aires, 1968, pp. 21-22), según la cual el sujeto sería transcendental, pero colectivo e inconsciente ("Kantismo sin sujeto transcendental»), y $2 .^{\circ}$ ) respecto a la crisis del humanismo. La afirmación: "El fin último de las ciencias humanas no es constituir al hombre, sino disolverlo" (El pensamiento salvaje, F.C.E., Méjico, 1964, p. 357), no podría ser nunca aceptada por Lacan, que aunque subvertido, mantiene al sujeto en tanto que fantasma fundamental o axioma del sujeto. El sujeto lacaniano es pura oscilación entre el pensamiento y el goce.

3 'El discurso de Roma' es el manifiesto del grupo disidente, que termina separándose de la S. P. P. (Sociedad Psicoanalítica de París) y fundando la S. F. P. (Sociedad Francesa de Psicoanálisis). Se incluye (Seuil, París, 1966) en los Escritos con el nombre de 'Función y campo de la palabra y del lenguaje en psicoanálisis'. En este momento aparece el axioma, que citaremos frecuentemente, que reza: «El inconsciente está estructurado como un lenguaje». 
psicoanálisis desde la invención freudiana del inconsciente, es un artificio destinado a construir un sujeto tachado irremediablemente, pero gozoso. Sujeto sujetado a un goce que le particulariza y le constituye en acto en tanto que pura diferencia.

Este artículo persigue distinguir entre el \$, sujeto tachado, y el fantasma $\$ \triangleright a$, que tal como puede apreciarse es la articulación del $\$$ a una letra, 'a', que Lacan denomina objet petit a (objeto 'a' minúscula), que designa el goce particular del sujeto universal del lenguaje. El artificio que denominamos psicoanálisis conduce al \$, en tanto que sujeto universal del lenguaje, a la construcción de su ser de sujeto del inconsciente. La construcción del fantasma $\$ \gg$ a, ventana hacia lo real, que sostiene el deseo del sujeto del inconsciente y asegura el goce del Otro, es el constructo que por la mediación del diseńo y la técnica psicoanalítica "transforma" al sujeto dividido por el significante en un sujeto del inconsciente: un sujeto producido por eso (ello) que habla en él.

El fantasma es, pues, un matema que expresa la articulación de un universal $\$ y$ un particular, 'a', que dice cómo gozar al sujeto, que lo orienta hacia un goce individual que lo constituye en tanto que sujeto sujetado al lenguaje, pero individualizado por su particular forma de gozar.

\section{Qué es el matema}

Si seguimos a Milner ${ }^{4}$ y distinguimos entre el primer y el segundo clasicismo de Lacan podemos separar la lógica del significante como perteneciente al primero y la topología del sujeto como perteneciente al segundo. Este segundo no tiene un manifiesto, 'El discurso de Roma', como ocurre con el primero, pero está la noción de matema, del griego "lo que se enseña», que marca con su aparición el corte entre ambos clasicismos.

Algo que persiste en Lacan es la matematización de los principios del psicoanálisis. Que esta matematización o formalización lógica, de sus principios se haga en dos etapas claramente diferenciadas, no significa sino que las insuficiencias de la lógica del significante le conducen a otro tipo de expresión lógico-formal: el matema.

En el primer clasicismo se adscribe a la formalización de la lengua. En este aspecto Lacan sigue a Stalin y sostiene la oposición entre la lengua como forma

4 MiLner, J. C.: L'oeuvre claire, Seuil, París, 1995. 
y la lengua como substancia. La lengua como forma es lo que la lingüística denomina estructura en tiempos de Stalin. Jakobson lo avala y Lacan se inspira. Pero también toma buena nota del formalismo matemático postulado por el grupo Bourbaki que intenta traducir las matemáticas en un cálculo lógico.

Que la matemática tiene el estatuto de una lengua es algo que acepta Bourbaki en tanto que lo que busca es su axiomatización. La evolución de la matemática la ha conducido a su literalización no cuantitativa y ello permite a Lacan el enunciado de su axioma primero: «El inconsciente está estructurado como un lenguaje" 5 .

En el segundo clasicismo, a partir de 1972, el matema o lo que se transmite sin resto, es decir, lo que es transmisible en su totalidad, es un enunciado conforme al paradigma matemático. Hay que insistir en el proyecto, ya apuntado, que mueve de principio a fin el intento de Lacan por ser lógico: 'reescribir el psicoanálisis «matemáticamente»", lo mismo que Bourbaki quería escribir axiomáticamente las matemáticas.

Todo lo dicho no significa sino que la diferencia entre ambos clasicismos está marcada no por el afán de Lacan de ser lógico, que se muestra como una constante de su trayectoria intelectual, sino por la distinción entre el significante, la pura diferencia anterior a toda cualidad, y la letra, identidad consigo misma, permutable en sí misma frente al significante que está íntegramente definido por el lugar que ocupa y es, por ello, indesplazable. A lo largo de este artículo nos proponemos explicar la afirmación lacaniana que reza: «La letra radicalmente es efecto de discurso" 6 .

Como el lector ya habrá supuesto, la letra efecto del discurso es la 'a' que aparece en la ¿̨órmula?, ¿matema? del fantasma, $\$ \triangleright$ a.

Después de establecer la diferencia entre los períodos anterior y posterior a 1972, año en que Lacan publica Encore y L'Étourdit, vamos a permitirnos usar el término de matema en un sentido amplio, tal como lo hacen la mayoría de

5 «El inconsciente está estructurado como un lenguaje» es un pleonasmo con el que Lacan intenta reforzar la identidad entre lenguaje y estructura. Corral, N.: El cortejo del mah Talasa, Madrid, 1996, p. 62.

Si Lacan, en un primer clasicismo, identifica lenguaje y estructura, en el segundo, siguiendo al grupo Bourbaki, identifica lenguaje y matemáticas, lo que le permite construir un sujeto topológico; la estructura, el tejido, del discurso psicoanalítico, es topológica.

6 LaCAN, J.: S. XX, Encore, Seuil, París, 1975, p. 36. La traducción es nuestra. 
los estudiosos de Lacan, de modo que lo haremos sinónimo de 'fórmula'. La fórmula del fantasma en su construcción topológica data de 1962 y, sin modificarla, aparece en L'Étourdit, ya citado. En los diez años transcurridos Lacan no modifica el matema del fantasma en tanto que constructo topológico o crosscap.

No obstante, en los diez años transcurridos entre 1962 y 1972, la evolución de la concepción lacaniana ha sufrido un corte epistemológico, se ha producido una torsión mayor que lleva a Lacan al abandono de la lógica del significante, de la lingüística estructural, para profundizar en la topología del sujeto, en la formalización y matematización de los principios del psicoanálisis.

\section{El sujeto tachado, $\mathbf{8}$}

Le sujet barré, el sujeto tachado o escindido por «una barra», es el objeto de la técnica y el diseño psicoanalítico en tanto que efecto de la estructura. Si el cero o vacío del sujeto viene a ser ocupado por el lenguaje, si el niño es nombrado antes de nacer y si el deseo de sus progenitores ya le ha dado una identidad imaginaria, un nombre que le sujeta al código, que le hace padecer del significante es que $\$$ muestra al sujeto en fading frente a keso que habla de él".

Este sujeto producido desde un lugar excéntrico a sí mismo es el 'parlanteser' (parlêtre), que puede buscar en la técnica y el diseño psicoanalítico un instrumento capaz de separarle de su alienación, estructural y estructurante, al Otro en tanto que tesoro del significante.

Hemos dicho puede, podríamos haber dicho si quiere, pero convendría recordar que Lacan, parafraseando a Freud, lo considera una exigencia. El imperativo es moral y la fórmula freudiana aparece numerosísimas veces en la obra de Lacan: Wo Es war soll Ich werden 7 . Dada la importancia de la transformación que debe operarse, el mandato que reza: "allí donde era Ello, allí yo debo advenir» exige la titánica, imposible labor de separación respecto al gran Otro que permita que «allí donde Ello habla, ahí me tengo que designar, ahí tengo que estar yo".

7 FREUD, S.: 'Las diferentes instancias de la personalidad psíquica', 1932, en Nuevas conferencias sobre psicoanálisis, G. W. XV, pp. 62-85. 
La tesis defendida por este artículo es que para Lacan la introducción del sujeto en lo social se hace "uno por uno" ${ }^{8}$, que el análisis en tanto que mediación para la resolución del Edipo es un rito de iniciación, un artificio que modifica al sujeto herido por el lenguaje. El sujeto construido por el psicoanálisis es un sujeto en el que se ha operado una modificación en su manera peculiar de gozar. El psicoanálisis es una técnica y un diseño que le reifican como detentador de un gozo, de una forma particular de goce que le otorga el ser-consistencia (étoffe) que le permite sostenerse frente a ese fading alienante que lo constituye.

Esta construcción del sujeto que le introduce al lazo social, que evita la enajenación entendida como falta de obra, como solipsismo empobrecedor cuyo máximo exponente es la "libertad" psicótica, es responsabilidad del sujeto. Tal como hemos dicho, hay una exigencia ética que invita, Lacan nos invita, a que cada uno rehaga el camino de la duda y haga suya la duda cartesiana que desemboca, si hay suerte y el proceso analítico llega a su término, con la certeza de la separación ${ }^{9}$, que es articulación de la pura diferencia en la red del discurso social.

Lacan ha usado el término 'sujeto' de forma analógica y nosotros pretendemos establecer las diferencias entre esos análogos. El \$ que nos ocupa es un sujeto originario y original, escindido y en fading, vacilante, frente al código que, a la vez que le constituye, le anula. El axioma según el cual rel sujeto es un significante para otro significante», hace de éste un sujeto representado, siendo su representante el amo que lo constituye, el significante que lo somete.

Esta alienación constituyente y estructural es superable, no respecto al lenguaje sino respecto a los significantes primeros a los que el sujeto quedó prendido. Separarse de estas insignias supone la apropiación del código, cuyo lugar es el campo del Otro, que cada sujeto debe llevar a cabo. El infans, retoño de la especie humana, "sale a buscar" su representante, su significante amo en el lugar de Otro. En ese lugar, que Lacan denomina el tesoro del significante, el sujeto es marcado por un significante, $S_{1}$, la insignia que gobernará su vida.

8 «La certitude n'est pas pour Descartes un moment qu'on puisse tenir pour adquis (...). Il faut qu'il soit, à chaque fois, par chacun, répété. C'est une ascèse. (...) C'est, à proprement parler, l'instauration de quelque chose de séparé". LACAN, J.: Seminario XI, Los cuatro conceptos fundamentales del psicoanálisis, Seuil, París, 1973, p. 204.

9 «Par la fonction de l'objet $a$, le sujet se sépare, cesse d'être lié à la vacillation de l'être, au sens qui fait l'essentiel de l'aliénation». LACAN, J.: Seminario XI, Los cuatro conceptos fundamentales del psicoanálisis, Seuil, París, 1973, p. 232. 


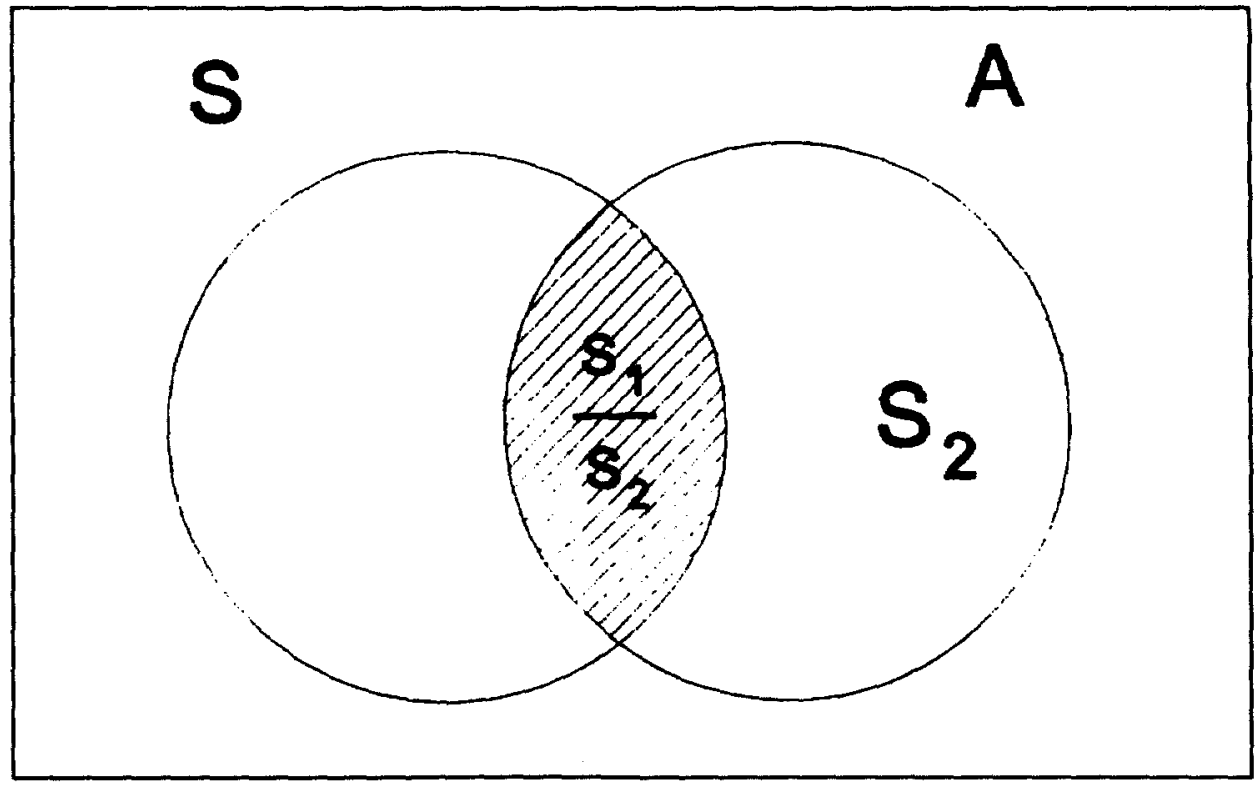

Así, en un tiempo inicial y que debemos considerar más desde su aspecto lógico que cronológico, el sujeto se encuentra a sí mismo marcado por algo que lo divide primordialmente de sí como sujeto de la palabra. En este nivel es en el que decimos que hay un sujeto tachado y vacilante frente al Otro significante. Pero este sujeto en suspenso, desvaneciente frente al Otro, tenderá a reificarse, a captarse a sí mismo y reconstituirse como sujeto de la palabra.

Si tomamos el Dictionnaire de la psychoanalyse ${ }^{10}$, podemos leer que $\$$ ues un efecto de la inmersión del retoño del hombre en el lenguajem. Nosotros vamos a añadir que los retońos crecen y, algunos, re-crecen con la mediación del artificio que propone el psicoanálisis.

El sujeto tachado por el significante y dividido en el pensamiento es el sujeto del deseo si y sólo si media la castración simbólica. El deseo es efecto de la castración, de la renuncia al objeto primordial. El sujeto tachado ha perdido tan maravilloso objeto, pero eso no significa que haya renunciado a él ${ }^{11}$. Si lo ha-

10 Réferences, Larousse Sciences Humaines, bajo la dirección de Roland Chemama, Larousse, París, 1993, p. 273.

11 La alienación estructural del sujeto al Otro puede resolverse espontáneamente y el sujeto quedará libre de la servidumbre a su madre, pero si esto no es así, la cura analítica puede ser el artificio adecuado para separar al 'parlanteser' del Otro primordial. 
ce, si renuncia via analitica a ese objeto inigualable, obtendrá una cierta ganancia, un plus-de-goce, que le constituye, es decir, que le hace ex-sistir.

Ese sujeto del deseo que también hemos denominado sujeto del inconsciente y que quizás fuera mejor matizar como sujeto del deseo inconsciente, indestructible como tal deseo, Freud dixit ${ }^{12}$, es el que Lacan sitúa en el fantasma $(\$ \diamond$ a) como efecto de respuesta al deseo del Otro. Para abordar el sujeto desde el fantasma es imprescindible pasar por el moi (Yo imaginario para Lacan y que traduce el yo como instancia freudiana opuesta al ello y al super yo), pero sin olvidar que este sujeto del inconsciente, menos sujeto que sujetado, no es un individuo biológico, ni un sujeto de la comprensión. El hecho de que el sujeto sea un mamífero no le reduce a ser un individuo biológico como el hecho de ser consciente no le reduce a la conciencia. En la Observación sobre el informe de Daniel Lagache ${ }^{13}$ Lacan marca la distancia entre la psicología y el psicoanálisis en tanto que más allá de la subjetividad se coloca el sujeto sin profundidades, pura superficie efecto del corte interpretativo.

Este sujeto del inconsciente tampoco es el yo de la gramática, no es el shifter de los lingüistas reducido a mero embrague de la enunciación. El sujeto del inconsciente es un axioma que se constituye en una ejecución. El sujeto es efecto del lenguaje en tanto ex-iste a partir de su propia enunciación. El sujeto se dice, se enuncia en forma de axioma. Este enunciado que denominamos axioma del sujeto es el fantasma. Para poder establecer este yo simbólico $(j e)$ es preciso pasar por el Yo imaginario $(m o i)$ que Lacan toma del yo de la segunda tópica freudiana.

\section{El moi o yo imaginario}

Tal como se ha explicado y representado mediante los círculos de Euler, el $\$$ producido en la relación del sujeto con el campo del Otro es un sujeto representado, en "ausencia", "por" un significante y "para" otro significante. Esta división del sujeto por el significante da cuenta de la finitud o, si se quiere, del

12 En la sesión del 29/1/1964, Seminario XI (Los cuatro conceptos fundamentales del psicoanálisis), Lacan se refiere a la interpretación de los sueños en relación con la certeza. Freud es cartesiano, Lacan dixit porque ha recorrido el camino desde la duda hasta la certeza. La certeza del sujeto del inconsciente es certeza sobre su deseo indestructible que emerge en lo evanescente del sueño.

13 Artículo de Lacan aparecido en los Escritos (Seuil, París, 1966) y que fue elaborado a partir del informe que presentó Lacan en el Coloquio de Royaumont (10-13/7/1958). 
hueco producido por la pérdida del objeto. El objeto perdido de Freud es un objeto mítico $y$, desde esa perspectiva, imaginario, pero también es simbólico y entonces la falta es ausencia de ser, falta estructural de plenitud ontológica que no habrá modo de remediar, pero sí de remedar. El objeto perdido por y para siempre jamás es la imposibilidad de una identidad acabada, de una completud entitativa que haría del sujeto un sí mismo, pero no implica que el sujeto no pueda "hacer como si» esa completud fuera posible. Tal completud no puede más que ser imaginaria, pero suple la carencia ontológica dando al sujeto una consistencia (étoffe) que orienta su goce. El tejido que da consistencia al sujeto del inconsciente es un entramado de goce, sostenido por una imagen (el fantasma); es decir, lo que da cuerpo al sujeto es una forma particular e intransferible de goce.

El objeto está perdido, pero se pueden recuperar, por mediación del diseño y la técnica psicoanalítica, sus puntos de referencia y obtener una cierta recuperacion, una cierta ganancia o un cierto plus-de-goce que hace ex-istir, aunque manteniéndose fuera del lenguaje, al desfalleciente sujeto del inconsciente.

La escisión del sujeto es la que nos permite establecer la distinción entre un Yo con mayúscula, correlato del moi étoffé francés que puede traducirse como 'persona henchida', es decir, «engordada» imaginariamente. Así, el moi del registro imaginario "da cuerpo" al yo con minúscula que en francés se dice je (en Freud es Ich) o sujeto del inconsciente, que es una pura línea, un borde, el borde único de una banda de Möbius. El sujeto sin profundidades, pura superficie que Lacan denomina je es opuesto al moi, proyección de esa superficie llamada $j$. Son opuestos en cuanto que uno es simbólico: sujeto formal y topológico, y el otro es un sujeto consistente imaginariamente, falsamente sustantivo, que se puede denominar yo sustancioso o henchido.

Lo que se pone de manifiesto es la dificultad para verter al castellano ${ }^{14}$ la diferencia entre estos dos yoes que en francés se pueden distinguir con facilidad como je/moi y que nosotros, una vez señaladas las diferencias entre ambos aspectos del sujeto escindido en y por el significante, vamos a citar en francés para mayor comodidad. Añadimos que estos dos yoes están en íntima relación

14 «La transliteración del Moi en 'Yo' (henchido en la majestad de la mayúscula) y el je en 'yo' (reducido a la imposible enunciación del sujeto del deseo inconsciente que lo deja vedado para el lenguaje) puede con todo resultar práctica». GARATE, I., y MarINAS, J. M.: Lacan en castellano, Quipú Ediciones, Madrid, 1996, p. 178. 
con el análisis del cogito cartesiano que Lacan desarrolla y que le conducen a la distinción entre el sujeto de la enunciación (inconsciente $=j e$ ) y sujeto del enunciado (consciente $=$ mo $i$ ).

\section{El fantasma: axioma del sujeto}

$\mathrm{Si}$, tal como se ha dicho, hay un sujeto o 'parlanteser' (parlêtre) desde el momento en que se produce la incidencia del significante sobre el retoño del hombre, eso no significa que ser parlante y sujeto del inconsciente se identifiquen. Muy al contrario, y tal como se dijo al principio, el sujeto del deseo inconsciente es un sujeto construido a partir de la técnica y el diseño psicoanalíticos.

El retoño del hombre, en tanto que sujeto, tiene en el significante su causa material y excéntrica, quedando así estructuralmente alienado al Otro en tanto que tesoro del significante. El Otro es el lugar donde el sujeto encuentra los significantes con los que decirse, aunque ese decirse imaginarizado sea fuente de desconocimiento y, lo que es más importante desde el punto de vista de la cura, de sufrimiento sintomático.

La metáfora sintomática pone en escena un goce cuyo significante amo, eje de la estructura o red significante subyacente, está reprimido. Desde este punto de vista, pues, hay un $\$$, un sujeto mortificado por el significante y alienado a los significantes de ese Otro primordial, la madre, donde el sujeto ha encontrado el lenguaje. La construcción del sujeto del inconsciente exigirá la separación de ese Otro primordial por mediación de la metáfora paterna. Que el Nombre del Padre surta efecto y la acción de la Ley sea eficaz puede ocurrir en el medio familiar del sujeto parlante, lo que excluiría el artificio que llamamos cura psicoanalítica. En caso de fracaso, el artificio psicoanalítico, mediante el corte interpretativo, separa al sujeto alienado de los significantes de ese Otro primordial para mejor alienarlo al código, único amo que hace que más que un sujeto del inconsciente tengamos que hablar de un sujeto sujetado al lenguaje.

Los axiomas lacanianos según los cuales: $\left.10^{\circ}\right)$ el inconsciente está estructurado como un lenguaje, y $2 .^{\circ}$ ) el significante representa a un sujeto ante otro significante, son los puntos de anclaje del je o sujeto del inconsciente en tanto que sujeto topológico. El sujeto del inconsciente es representado por Lacan en 1962, en la sesión del 6/6/1962 de su Seminario sobre L'identification como cross-cap. Este objeto topológico es equivalente al plano proyectivo y puede separarse en otros dos objetos. 
El primero es la banda de Möbius, ya citada, que es una cinta sin orientación, reductible a la línea de su único borde, y con la que Lacan representa al sujeto tachado \$. El sujeto escindido por el significante es un sujeto vacilante, en fading frente al significante que lo designa, anulándolo, y sin orientación alguna. Podría comparársele a un guiñol. El \$ es el sujeto universal del lenguaje $\mathrm{y}$, desde ese punto de vista, un genérico que exige algo que lo particularice.

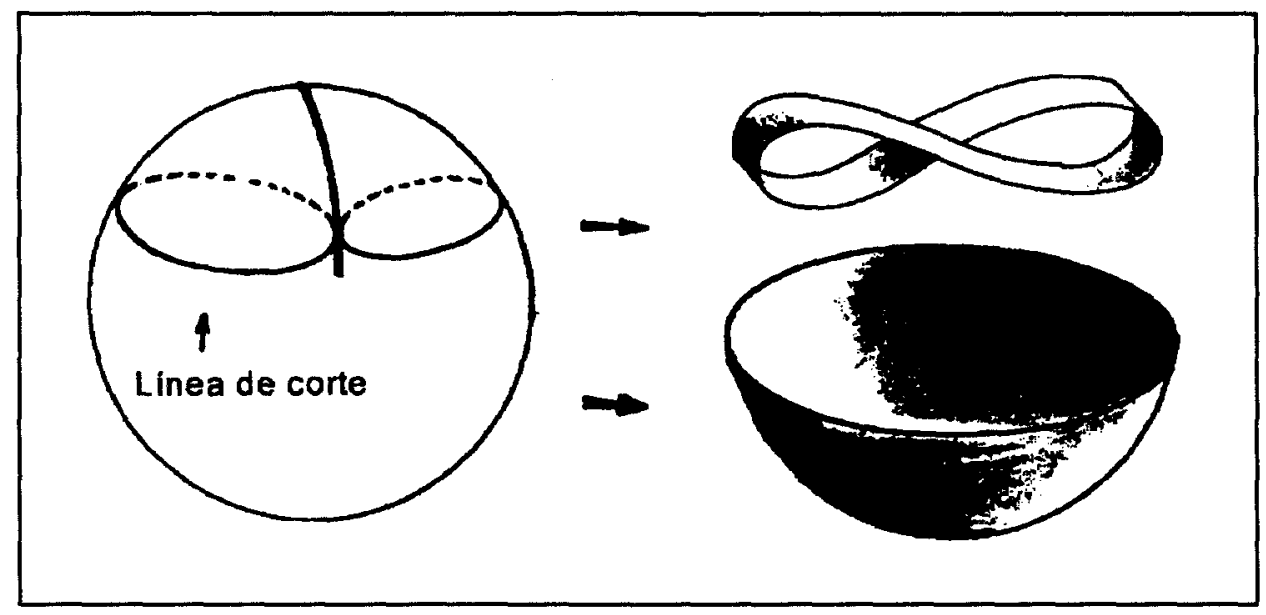

El segundo es el objeto que individualiza al sujeto dividido por el significante. Este objeto, el objeto 'a', es un disco que bordea la línea dándole orientación y consistencia de goce. El disco es el objeto 'a', plus-de-goce o ganancia de goce que se adquiere por la renuncia al objeto primordial. La castración simbólica no es otra cosa que la renuncia mediante la eficacia del Nombre del Padre, que introduce al sujeto a la ley del deseo inconsciente.

El sujeto del inconsciente es un sujeto que desea y goza más allá de ese objeto primordial porque en lugar de estar fijado a los significantes insignia de su primera infancia ha quedado sujetado al lenguaje. Se trata, pues, de una nueva alienación dado que el sujeto del inconsciente está cautivo en el código, en el que se pierde para poder ser representado por los significantes. Estos significantes son metonímicos, el objeto del deseo también lo es y, por tanto, sus representantes son sustitutos que le representan en el discurso que constituye su vida de sujeto. 
La fórmula del fantasma fundamental, $\$ \triangleright$ a, que Lacan define como crosscap en 1962, vuelve a ser presentada como matema diez años después. En L'Étourdit, publicado en 1972, Lacan continúa definiendo al sujeto del inconsciente o je como cross-cap divisible en la articulación, superadora de la alienación originaria, de sus dos componentes: el sujeto $\$$ y el objeto 'a'. El losange, $\diamond$, signo de la articulación, puede designar tanto la alienación, o intersección $(\Lambda)$ del $\$$ al Otro, como su separación (disyunción, $\underline{V}$ ) efectuada por el corte interpretativo. La interpretación que el analista realiza, una vez escuchados los dichos del analizante, es un corte, semejante al que hacen los topólogos en sus objetos, y que nosotros podríamos reproducir con papel y tijeras. Este corte efectuado sobre el sujeto en tanto que cross-cap hace que el sujeto modifique su estructura sin salir de ella. El corte interpretativo articula una banda sin orientación con un disco orientador que dirige al sujeto, deseante, hacia una forma de goce que le particulariza. Este goce da consistencia al sujeto evanescente, le da cuerpo y nombre propio.

\section{La subversión del cogito cartesiano}

Lacan establece la escisión del sujeto a partir del análisis del cogito cartesiano al que subvierte dado que, según la relectura lacaniana, lo que el cogito funda no es la conciencia sino el sujeto tachado o \$. De esta forma Lacan puede darle un asiento al sujeto del deseo inconsciente del que habla Freud ${ }^{15}$.

En el año 1967 Lacan deduce el sujeto freudiano del cogito cartesiano, lo que le conduce a definir el sujeto del inconsciente como fantasma fundamental o definición axiomática en la que el sujeto funda su propio discurso.

El desidero freudiano es identificado desde 1964 con el cogito cartesiano, pero en La lógica del fantasma da un paso más al utilizar la negación bajo la formulación que de ella hace el lógico Morgan al establecer la regla de la equivalencia entre la negación de la conjunción de dos proposiciones y la disyunción de la negación de cada una de las proposiciones intervinientes .

15 Este artículo no hace un recorrido por las distintas relecturas que Lacan hizo del cogito cartesiano. Sólo vamos a referirnos a la reinterpretación del Seminario de 1966/1967, titulado La lógica del fantasma. Para seguir el discurso lacaniano sobre la subversión del sujeto se deben consultar los Seminarios de los cursos 1963/1964 y 1965/1966. 
La aplicación de las leyes de Morgan al cogito cartesiano transforma la formulación condicional: cogito ergo sum, que habría que estudiar como una implicación material, en la negación de una conjunción: es imposible para el sujeto, dice Lacan, pensar (inconsciente) y ser simultáneamente. Lo que se convierte, al aplicar las leyes de Morgan, en: «o yo no pienso o yo no soy».

Esta disyunción permite a Lacan colocar el "Yo no soy" del sujeto freudiano del inconsciente en relación con el "yo pienso" cartesiano.

Si el cogito es coextensivo a la racionalidad inconsciente, la escena metafisica cartesiana sería inconsistente. La escena metafisica no procedería del discurso consciente sino del deseo inconsciente de existir en tanto que se piensa. Pero si el cogito es producto del inconsciente estructurado como un lenguaje, éste en tanto que escena metafisica queda reducido a un fantasma, es el producto fantasmático de Descartes en tanto que moi étoffé.

Tal como queda expresado parece que Lacan ha culminado con éxito su intento de dotar a Freud de un sujeto ${ }^{16}$ del deseo inconsciente. Freud sólo dice que sus pacientes están alienados a una escena inconsciente. Esta escena inconsciente es llamada por Freud 'fantasma' 17 y es revalorizada por Lacan, hasta constituir, como veremos, la identificación del sujeto del deseo inconsciente con el fantasma fundamental efecto de la castración simbólica.

16 La lectura que Lacan hace de Freud consiste primeramente en una crítica del naturalismo freudiano. Lacan se vuelve hacia Descartes para buscar un sujeto que soporte el indestructible deseo freudiano, pero para sustituir la referencia naturalista necesita sustituir las referencias biológicas - sustitución, por ejemplo, del instinto por la pulsión articulada con el Otro del lenguaje - por la noción de estructura. Es el estructuralismo el que dota a Lacan del instrumento que le permite sistematizar la articulación, que ya estaba presente en Freud, de la sexualidad con la estructura del lenguaje.

Lo que aquí nos interesa destacar es que en las condiciones marcadas por Lacan al introducir el sujeto, por más inconsciente que sea éste, ya no le es posible tener una relación inmediata con la sexualidad. El sujeto no puede tener relación con la sexualidad sino por el sesgo que la estructura del lenguaje le permite. De ahí que lo real de la sexualidad, eso que procede de nuestra condición de mamíferos, no pueda ser abordada nada más, y nada menos, que por los registros simbólico e imaginario. La escena metafísica del cogito cartesiano queda reducida a una fantasía que muestra no sólo la alienación del sujeto al Otro del lenguaje, sino también lo más importante desde nuestra perspectiva filosófica: el déficit ontológico que se vela con la articulación simbólico-imaginaria que proporciona el fantasma. No en balde la formulación del fantasma está hecha por el yo henchido (moi étoffe) que la cura analítica "pincha" para designar el agujero entitativo que encubre.

17 Lacan revaloriza la noción de fantasma a partir del artículo, escrito por Freud en 1919 , titulado 'Pegan a un niño'. 
El fantasma fundamental está producido, como se ha dicho, por el corte interpretativo del analista que efectúa la separación entendida en términos de castración simbólica. No hay separación de la alienación al Otro primordial sin la mediación del operador fálico que llamamos Nombre del Padre o Ley simbólica que prohíbe el incesto y separa de los significantes que fueron la prehistoria del sujeto, pero que lo introduce en el lenguaje en tanto que escindido por el significante y articulado a un resto de goce, plus-de-goce o ganancia de goce efecto del análisis. Este goce recuperado viene a colocarse en el lugar del objeto perdido.

El sujeto ha renunciado a ser un objeto fálico para el Otro y esta pérdida le produce un vacío que no se puede colmar jamás pero que le hace desear (inconscientemente). El deseo se orienta hacia una forma personalísima de goce que hace ex-sistir al sujeto tachado por el código. Ese punto de goce, que Lacan llama objeto 'a', lastra al sujeto que cual marioneta (la banda de Möbius carece de orientación) seguiría dando bandazos bajo los efectos del significante que a la vez que lo designa como sujeto lo anula bajo sus efectos mortificantes.

Pero, para que todo esto ocurra debe mediar el artificio psicoanalítico que efectúa la castración simbólica del sujeto y le constituye como fantasma fundamental o $j$ e. El je se enuncia a sí mismo, se dice en forma de axioma del sujeto, después del largo viaje que es la cura analítica. Este viaje podemos calificarlo como el paso del síntoma al fantasma. Y este viaje no es más que el paso desde los fantasmas inconscientes ${ }^{18}$, producidos por la alienación al Otro del lenguaje, hasta ese axioma del sujeto, que va a constituir su fantasma fundamental al poder articularse el $\$$ original con una forma particular de goce, 'a'.

La disyunción que Lacan establece en La lógica del fantasma permite establecer la alternancia entre los dos elementos del fantasma, $\$ \triangleright$ a, entre el $\$$ en el campo del Otro y el objeto 'a', la pulsión o el "eso piensa" pulsionalmente.

Según la regla de la disyunción, si "yo no pienso" es verdadero, sería falso afirmar que «yo no existom. Existo en tanto que la pulsión actúa y goza. El objeto 'a' del matema del fantasma es un plus-de-goce, una ganancia de goce que se produce cuando el ello freudiano, la reserva pulsional, se pone en marcha. El

18 Fantasmas que se activan y se escenifican en la vida del sujeto sin que éste sepa de dónde proceden esos síntomas que se encarnan en él y le mortifican. Rota la conexión ente la escena inconsciente y los lapsus, sueños y demás formaciones del inconsciente, el sujeto $\$$ sólo vive su aplastamiento por el significante. 
ser del sujeto, su existencia, no procede, pues, de la conciencia: no soy un yo consciente sino un eso pulsional que goza.

Cuando el "yo no existo" es verdadero es imposible afirmar con verdad que "yo no pienso". El \$ piensa desde el campo del Otro, desde el inconsciente, lugar del tesoro significante. Pero seguimos estando fuera de la conciencia y en el campo del inconsciente estructurado como un lenguaje que hace del sujeto un puro representado por el significante.

Esta alternancia, verdadera oscilación entre los dos polos del matema, debe desembocar en una formulación axiomática del sujeto que pone en acto la exigencia freudiana; este "yo no pienso", en tanto que correlato del ello, está llamado a articularse con el "yo no soy", en tanto que correlato del inconsciente, pero de una manera que en cierto modo se eclipsan, se ocultan el uno al otro recubriéndose. Lacan ${ }^{19}$ dice textualmente: "Es al lugar del "yo no soy" que el ello debe advenir, positivándolo en "yo soy eso" que no es más que el puro imperativo, un imperativo que no es otro que el formulado por Freud como Wo Es war, soll Ich werdens.

"Eso que yo soy» es una forma de goce, un ser pulsional que me da consistencia corporeizándome. El sujeto del deseo inconsciente se nombra, se dice como objeto pulsional. El fantasma como axioma del sujeto no es otra cosa que una exigencia lógica procedente de que los objetos pulsionales concretos: oral, anal, genital, no pueden expresarse fuera de las reglas gramaticales. Aunque sea eso lo que habla de mí en lugar de ser yo el que habla, las reglas gramaticales son las mismas y Freud las dejó establecidas: 'devorar/ser devorado/hacerse devorar' serán siempre las formas de expresión de un fantasma oral como el 'agredir/agredirse/hacerse agredir' lo serán de un fantasma sadomasoquista. En estos ejemplos el fantasma está reducido a la pulsión tal como ocurre en el final del análisis donde el sujeto se reduce a una forma personal e intransferible de goce que lo particulariza y lo distingue del universal sujeto del lenguaje, $\$$, que ha sido y seguirá siendo, aunque bordeado y sostenido por un alter ego de gozo.

La formulación del axioma del sujeto Descartes ha sido finalmente considerada por Hintikka como una performance, es decir, como una ejecución. Desde 1962 Hintikka ${ }^{20}$ estudia el cogito cartesiano a fin de discriminar si es una in-

19 Lacan, J.: La lógica del fantasma, Seminario inédito; sesión del 11/1/1967. La traducción es nuestra.

20 Hintikka, J.: "Cogito ergo sum: inférence ou performance?», Philosophical Review, vol. LXXI, enero de 1962. 
ferencia o una ejecución. Parece que en el número de 1996 de la Revista Internacional de Filosofia, Hintikka se decide finalmente a considerar que el cogito cartesiano no es una inferencia sino una performance. De este modo, y tal como lo plantea Lacan, es una decisión del sujeto, es una ejecución del sujeto: el sujeto Descartes existe en tanto que se enuncia como pensante, mejor que como vidente o cantante.

Ello no significa que no haya una argumentación previa que le conduzca hacia esa resolución y no a otra, pero, y en ello reside la diferencia con la implicación material, no hay un nexo apodíctico entre la argumentación previa y la conclusión. La conexión de necesidad es un imperativo lógico-formal que impide cortar o dejar en suspenso, por decisión consciente o inconsciente, la conclusión correspondiente. No se trata, pues, de inferencia en el cogito cartesiano, sino de una decisión del sujeto Descartes que se define a sí mismo, tras una argumentación previa, como existente en y por el pensamiento.

Para terminar, es necesario volver al primer apartado de este artículo y a la distinción, tomada de Milner, entre el primer y el segundo clasicismo lacaniano. La distinción entre ambos se funda en la torsión efectuada por Lacan al pasar de la lógica del significante a la topología del sujeto. Quizás ahora la torsión se muestre como necesaria. Lacan quería ser lógico en el sentido de dotar al psicoanálisis de unos principios científicos y comenzó por servirse de los instrumentos que la lógica y la lingüística le proporcionaron, pero la insuficiencia de ambas para proporcionarle los medios que le condujeran a la construcción de un sujeto que no se dedujera apodícticamente, al más puro estilo del racionalismo moderno, le condujo hacia la topología, que no usa de la demostración sino de los conceptos ${ }^{21}$ de adyacencia, vecindad y disección de los cuerpos para construir sus objetos.

Si $\$$ es el sujeto escindido por los significantes del Otro con los que en vano intenta contestarse a las cuestiones clave: ¿qué quiere el Otro?, ¿qué quiero yo?, ¿qué soy yo?, será necesario pasar del significante, que es la pura diferencia, a otro elemento que permita parar la metonimia que le es propia. Un significante siempre puede ser sustituido por otro, pero esto no es así con la letra que tiene cualidades y que sólo puede venir a ocupar el lugar que le corresponde dentro del álgebra. La asociación libre permite pasar de un significante a otro 
hasta que se produce una significación que inmoviliza la metonimia significante. En este momento de la congelación se ha producido la letra como efecto del discurso analítico: la 'a' minúscula, efecto del discurso, viene a ocupar el lugar del objeto perdido para siempre (el seno), objeto que causa el deseo en tanto que deja un vacio que hay tendencia a colmar. En ese lugar viene la letra, significante congelado en su significación, como recuperación de goce que da corporeidad al sujeto. 